\title{
Does Not Know
}

National Cancer Institute

\section{Source}

National Cancer Institute. Does Not Know. NCI Thesaurus. Code C67142.

The answer is not known by the person answering. 